\title{
Nuclear quantum effects in state-selective scattering from ring polymer molecular dynamics
}

Cite as: J. Chem. Phys. 152, 194113 (2020); https://doi.org/10.1063/5.0004179

Submitted: 08 February 2020 . Accepted: 01 May 2020 . Published Online: 21 May 2020

Adrien Marjollet (iD, and Ralph Welsch (iD)
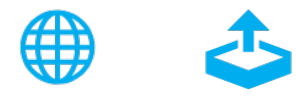

\section{ARTICLES YOU MAY BE INTERESTED IN}

Essentials of relativistic quantum chemistry

The Journal of Chemical Physics 152, 180901 (2020); https://doi.org/10.1063/5.0008432

Assignment of vibrational states within configuration interaction calculations

The Journal of Chemical Physics 152, 194112 (2020); https://doi.org/10.1063/5.0009732

PSI4 1.4: Open-source software for high-throughput quantum chemistry

The Journal of Chemical Physics 152, 184108 (2020); https://doi.org/10.1063/5.0006002

\section{Lock-in Amplifiers up to $600 \mathrm{MHz}$}
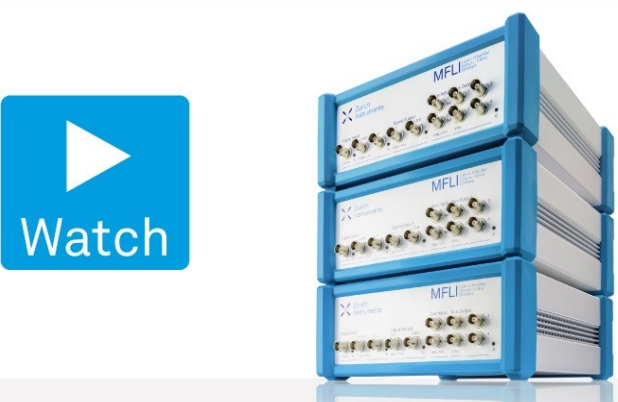


\title{
Nuclear quantum effects in state-selective scattering from ring polymer molecular dynamics
}

\author{
Cite as: J. Chem. Phys. 152, 194113 (2020); doi: 10.1063/5.0004179 \\ Submitted: 8 February 2020 - Accepted: 1 May 2020 • \\ Published Online: 21 May 2020
}

Adrien Marjollet ${ }^{1,2}$ and Ralph Welsch ${ }^{1,3, a)}$ iD
AFFILIATIONS
${ }^{1}$ Center for Free-Electron Laser Science, DESY, Notkestrasse 85, 22607 Hamburg, Germany
${ }^{2}$ Department of Physics, Universität Hamburg, Jungiusstrasse 9, 20355 Hamburg, Germany
${ }^{3}$ The Hamburg Centre for Ultrafast Imaging, Luruper Chaussee 149, 22761 Hamburg, Germany

a) Author to whom correspondence should be addressed: ralph.welsch@desy.de

\begin{abstract}
We present an efficient method to obtain initial state-selective cross sections for bimolecular reactions that can account for certain nuclear quantum effects by employing the ring polymer molecular dynamics approach. The method combines the well known quasiclassical trajectory (QCT) approach with the description of the system in an extended ring polymer phase space. Employing the prototypical $\mathrm{Mu} / \mathrm{H} / \mathrm{D}+\mathrm{H} 2$ $(v=0,1)$ reactions as a benchmark, we show that the presented approach does not violate zero-point energy constraints and that it can also capture the contributions of tunneling through the $v=1$ vibrationally adiabatic barrier present for the $\mathrm{Mu}+\mathrm{H}_{2}(v=1)$ reaction. This is a significant improvement over the QCT approach with only a small increase in numerical cost.
\end{abstract}

Published under license by AIP Publishing. https://doi.org/10.1063/5.0004179

\section{INTRODUCTION}

Bimolecular reactions in the gas phase or chemisorption of a molecule onto a surface are important classes of reactions with relevance to many areas of chemistry, e.g., atmospheric and interstellar chemistry, combustion, and catalysis. ${ }^{1-5}$ State-selective measurements and simulations of these processes provide a detailed understanding of the reaction dynamics and help us understand the flow of energy in chemical reactions. The detailed understanding of the reaction dynamics can lead to efficient ways for controlling and steering these reactions. While impressive measurements can already provide detailed information for polyatomic systems, ${ }^{1,2,6-13}$ a full-dimensional quantum-dynamical description of state-selective scattering remains a great challenge. The biggest system treated today is the $\mathrm{H}+\mathrm{CH}_{4} \rightarrow \mathrm{H}_{2}+\mathrm{CH}_{3}$ reaction, ${ }^{4-20}$ which was simulated employing the quantum transition state concept ${ }^{21-27}$ and the multi-configurational time-dependent Hartree approach ${ }^{28-35}$ for the wavepacket propagation. However, these simulations are very involved and intricate; require many prerequisites, e.g., a fitted potential energy surface (PES) that can be evaluated efficiently ${ }^{36,37}$ and an appropriate curvilinear coordinate system; ${ }^{38}$ and take up many months of simulation time for each specific reaction.

Therefore, most reactive scattering simulations employ the quasiclassical trajectory (QCT) approach. ${ }^{39-41}$ In this approach, all nuclei are treated classically and evolve on an accurate PES or are based on gradients obtained from on-the-fly ab initio calculations. ${ }^{42}$ Suitable initial conditions for each trajectory are chosen to include the correct amount of zero-point energy ( $\mathrm{ZPE}$ ) and the correct amount of additional energy for a vibrational (or rotational) excitation. This approach has been very successful for the description of bimolecular and gas-surface reactions, in particular for energies well above the threshold. A great advantage of the QCT approach is its numerical efficiency, as only Newton's equations of motion have to be solved. This allows for the treatment of large systems, and it is even possible to perform QCT simulations while evaluating the potential energy and gradients on-the-fly. However, QCT simulations suffer from the ZPE leakage problem and do not respect ZPE constraints of the products. Due to the classical nature of the simulation, any ZPE put into a certain degree of freedom initially can artificially leak out into other degrees of freedom, in particular the 
reaction coordinate, and help overcome the barrier. Additionally, in QCT, the products can violate ZPE constraints by having less energy than the ZPE would require, which can lead to many additional reactive trajectories. Both these problems can lead to an increased reactivity in QCT compared to quantum simulation. ${ }^{43}$ Furthermore, QCT simulations do not include tunneling effects, which can lead to underestimation of the cross section, in particular close to the threshold energy and in systems where protons are transferred as part of the reaction. ${ }^{44}$ These problems can be manifest in a single reaction when looking at the reactivity of different initial vibrational states.

Various methods have emerged that approximately include nuclear quantum effects in classical-like simulations. Among the most used ones is the ring polymer molecular dynamics (RPMD) approach. ${ }^{45-48}$ RPMD is based on the imaginary-time path-integral formalism that maps a quantum Boltzmann distribution onto a set of classical replicas in phase space joined by harmonic springs, which is known as ring polymer. Dynamical quantities, such as real-time correlation functions, are obtained from classical dynamics in this extended ring polymer phase space. RPMD is exact in the hightemperature, short-time, and harmonic limits. It incorporates ZPE effects and does not suffer from ZPE leakage. ${ }^{4-51}$ Furthermore, it can incorporate some tunneling effects due to the extension of the ring polymer that can stretch over a barrier and thus lower the effective barrier height. ${ }^{52}$ RPMD has been especially successful for the calculations of thermal rate constants in the form of RPMD rate theory. ${ }^{47,48,52-54}$ RPMD rate theory is particularly efficient as it allows for the calculation of the thermal rate constant based on dynamics around the barrier region without the need to resolve the initial or final ro-vibrational states. Employing RPMD rate theory to various isotopologues of the $\mathrm{H}+\mathrm{H}_{2}$ reaction, it was impressively shown that it incorporates ZPE effects as well as tunneling. ${ }^{51,52}$ The original RPMD formulation is restricted to the simulation of equilibrium correlation functions. Recently, it was shown that RPMD can be effectively used to calculate correlation functions and expectation values associated with non-equilibrium initial conditions, e.g., for the case of an initial momentum kick. ${ }^{55}$ As the RPMD approach with equilibrium and non-equilibrium initial conditions can be obtained from Matsubara dynamics ${ }^{56}$ in similar ways, both exhibit the same well-known properties, e.g., it being exact in the high-temperature and classical limits, for dynamics in harmonic potentials and conserving the average energy of the springs. ${ }^{55}$ Further numerical tests showed that one can expect similar accuracy of RPMD for calculations with equilibrium and non-equilibrium initial conditions. ${ }^{5 .}$ Very recently, the non-equilibrium initial conditions have been used to obtain microcanonical rate constants from RPMD simulations; ${ }^{50,57}$ however, initial state-selectivity could not be addressed in these works.

In this manuscript, we describe an efficient approach to combine QCT simulations with the RPMD approach to obtain initial state-selective cross sections for bimolecular and molecule-surface reactions, which include several non-classical effects. These advantages of the method are exemplified using isotopic variants of the $\mathrm{H}+\mathrm{H}_{2}$ reaction. In particular, the $\mathrm{Mu}+\mathrm{H}_{2}$ reaction serves as a benchmark as it highlights both the missing ZPE constraints and the missing tunneling contributions in QCT simulations. ${ }^{44}$ The RPMD approach respects ZPE constraints, and based on RPMD simulations of non-reactive polyatomic systems, we expect that it will not suffer from ZPE leakage. Furthermore, the method only slightly overestimates the exact reactive cross section for $\mathrm{Mu}+\mathrm{H}_{2}(v=1)$, where tunneling through the $v=1$ adiabatic barrier is present. ${ }^{44}$ Section II describes the details of the method. Section III presents the results obtained for $\mathrm{Mu} / \mathrm{H} / \mathrm{D}+\mathrm{H}_{2}(v=0,1)$. Conclusions and an outlook are given in Sec. IV.

\section{METHOD}

To perform state-selective scattering calculations within the RPMD approach, we follow the idea of quasiclassical trajectories (QCTs). For illustrative purposes, let us consider an atom + diatom reaction $\mathrm{A}+\mathrm{BC}$. The generalization to polyatomic reactions is discussed further below. For the initialization of the diatomic molecule $\mathrm{BC}$, we proceed in two steps, first we employ the harmonic approximation with $\omega$ as the vibrational frequency of $B C$ to sample the initial ring polymer configurations for the vibrational normal mode of $\mathrm{BC}$ from the well-known Boltzmann distribution of a harmonic system. Corresponding Cartesian coordinates for the beads are obtained by inverse normal mode transformation. Sampling the normal mode guarantees that the molecule will not undergo spurious rotations or center of mass motion. These configurations represent a system containing the ZPE with additional thermal energy. By employing the path integral approach, we obtain correct initial quantum fluctuations for the position and momentum normal mode of BC. To obtain reliable scattering results, $\beta=\frac{1}{k_{B} T}$ has to be chosen high enough in order to make the contribution of the additional thermal energy to the reaction dynamics negligible. The choice of $\beta$ is discussed below.

Vibrationally excited states are mimicked by modifying the initial position and momentum of the ring polymer centroids, $\bar{Q}, \bar{P}$, to add the correct amount of vibrational energy since the initial sampling already contains the correct amount of ZPE. This step is again inspired by the QCT approach. To this end, we add to the mass scaled positions and momentum centroid of the ring polymer representing the vibrational mode of $\mathrm{BC}$ the following quantities:

$$
K_{Q}=\sqrt{\frac{2 v}{\omega}} \cos (\phi), \quad K_{P}=\sqrt{2 v \omega} \sin (\phi),
$$

where $\phi \in[0,2 \pi]$ is a random phase and $v$ is the vibrational quantum number. Following this step, the mass scaled position and momentum fluctuations for each bead $k \in[1, n]$ are

$$
\left\langle Q_{v}^{2}\right\rangle_{k}=\frac{1}{2 \omega} \operatorname{coth}\left(\frac{\beta \omega}{2}\right)+\frac{v}{\omega}, \quad\left\langle P_{v}^{2}\right\rangle_{k}=\frac{\omega}{2} \operatorname{coth}\left(\frac{\beta \omega}{2}\right)+v \omega .
$$

The initial ring polymer configurations of $\mathrm{BC}$ are then used in the scattering calculations. To this end, we directly switch to the full PES. In order to avoid extra fictitious vibrational energy introduced by the direct switching, we discard configurations with interatomic distances smaller than $d_{\min }$. $d_{\min }$ can be chosen based on the initial distances of vibrationally excited BC in QCT simulations. Upon switching, we have good stability of BC's internal energy around its harmonic energy value $\omega\left(v+\frac{1}{2}\right)$. This constitutes a reliable and consistent approximation in the case of a diatomic molecule with reasonable masses. This approach can be generalized to any polyatomic molecule by employing the normal modes of the respective 
molecule. Finally, the initial relative centroid velocities of $\mathrm{BC}$ are "kicked" toward A along the $\mathrm{x}$ axis,

$$
\overline{\boldsymbol{V}}_{B}=\overline{\boldsymbol{V}}_{C}=-\frac{\boldsymbol{P}_{r e l}}{m_{B}+m_{C}},
$$

where $m_{B}$ and $m_{C}$ are the masses of B and C, respectively, and the relative momentum set is $\boldsymbol{P}_{\text {rel }}=\sqrt{2 \mu E_{\mathrm{col}}} \boldsymbol{\kappa}$, where $E_{\mathrm{col}}$ and $\mu$ are the collision energy and the reduced mass of the system, respectively, and $\boldsymbol{\kappa}$ is the unit vector along the $\mathrm{x}$ axis.

Atom $\mathrm{A}$ is initialized far away from $\mathrm{BC}$ with the distance of the centroid of $\mathrm{A}$ to the centroid of the center of mass of $\mathrm{BC}$ equaling

$$
d(A, B C)=d(A, B C)_{\mathrm{QCT}}+R_{A}^{g}+\max \left(R_{B}^{g}, R_{C}^{g}\right),
$$

where $d(A, B C)_{\mathrm{QCT}}$ is the distance that would be used for standard QCT simulations and $R_{i}^{g}, i=A, B, C$ is the radius of gyration of the ring polymer for atoms $\mathrm{A}, \mathrm{B}$, and $\mathrm{C}$, respectively. The centroid of the ring polymer representing atom $\mathrm{A}$ is then displaced away from the molecular axis of $\mathrm{H}_{2}$ according to the impact parameter $b$ as in QCT. The initial centroid velocity of $A$ is set to

$$
\overline{\boldsymbol{V}}_{A}=\frac{\boldsymbol{P}_{r e l}}{m_{A}} .
$$

The initial non-centroid modes of the ring polymer for atom A are drawn from a free ring polymer distribution along the direction of propagation $\boldsymbol{\kappa}$.

Integral cross sections for a given total energy $E_{\mathrm{tot}}=E_{\mathrm{col}}+\omega(v$ $+\frac{1}{2}$ ) are obtained following the QCT approach as

$$
\sigma\left(E_{\text {tot }}\right)=\pi b_{\max }^{2} \frac{N_{R}\left(E_{\mathrm{tot}}\right)}{N\left(E_{\mathrm{tot}}\right)},
$$

where $N\left(E_{\mathrm{tot}}\right)$ and $N_{R}\left(E_{\mathrm{tot}}\right)$ are the number of total and reactive trajectories, respectively, and $b_{\max }$ is chosen so that no reaction occurs for $b>b_{\max }{ }^{41}$ The number of reactive trajectories is obtained by evaluating suitable distance criteria that distinguish the products.

$\beta$ is set depending on the initially chosen collision energy $\left(E_{\mathrm{col}}\right)$ and the energy added to $\mathrm{BC}$ by our vibrational excitation scheme $\left(E_{v}\right.$ $=\omega v)$ as

$$
\beta=\frac{2\left(\frac{m_{\mathrm{tot}}}{\mu}-2\right)}{\pi\left(E_{c}+E_{v}\right)},
$$

where $m_{\text {tot }}$ is the total mass of the system. The above choice of $\beta$ corresponds to an average of $\beta_{\mathrm{A}}$ and $\beta_{\mathrm{BC}}$. Here, $\beta_{\mathrm{A}}$ and $\beta_{\mathrm{BC}}$ are chosen such that the average relative velocities of $\mathrm{A}$ and $\mathrm{BC}$ match the corresponding QCT values in the center of mass reference frame. For higher collision energies, this can lead to low values of $\beta$, resulting in non-negligible effects due to the additional thermal energy. Therefore, we set a lower cutoff value so that $\beta=\beta_{-}$if $\beta<\beta_{-}$. A more detailed discussion of the choice and further tests of the robustness are beyond the scope of this manuscript and will be part of a forthcoming publication. Possible generalizations of the approach are discussed at the end of this manuscript.

\section{RESULTS}

As a benchmark system, we choose the triatomic reaction of $\mathrm{Mu} / \mathrm{H} / \mathrm{D}+\mathrm{H}_{2}(v=0,1)$. In particular, the reaction of $\mathrm{Mu}+\mathrm{H}_{2}$ exemplifies the problems of QCT simulations. For the reaction of $\mathrm{Mu}$ with $\mathrm{H}_{2}$ in its ground vibrational state, the ZPE constraint problem is dominant and QCT simulations find a threshold of about $0.2 \mathrm{eV}$ lower than exact quantum dynamics simulations. ${ }^{44}$ Yet, for the reaction of $\mathrm{Mu}$ with vibrationally excited $\mathrm{H}_{2}$, QCT results find a threshold of about $0.15 \mathrm{eV}$ higher than exact quantum dynamics
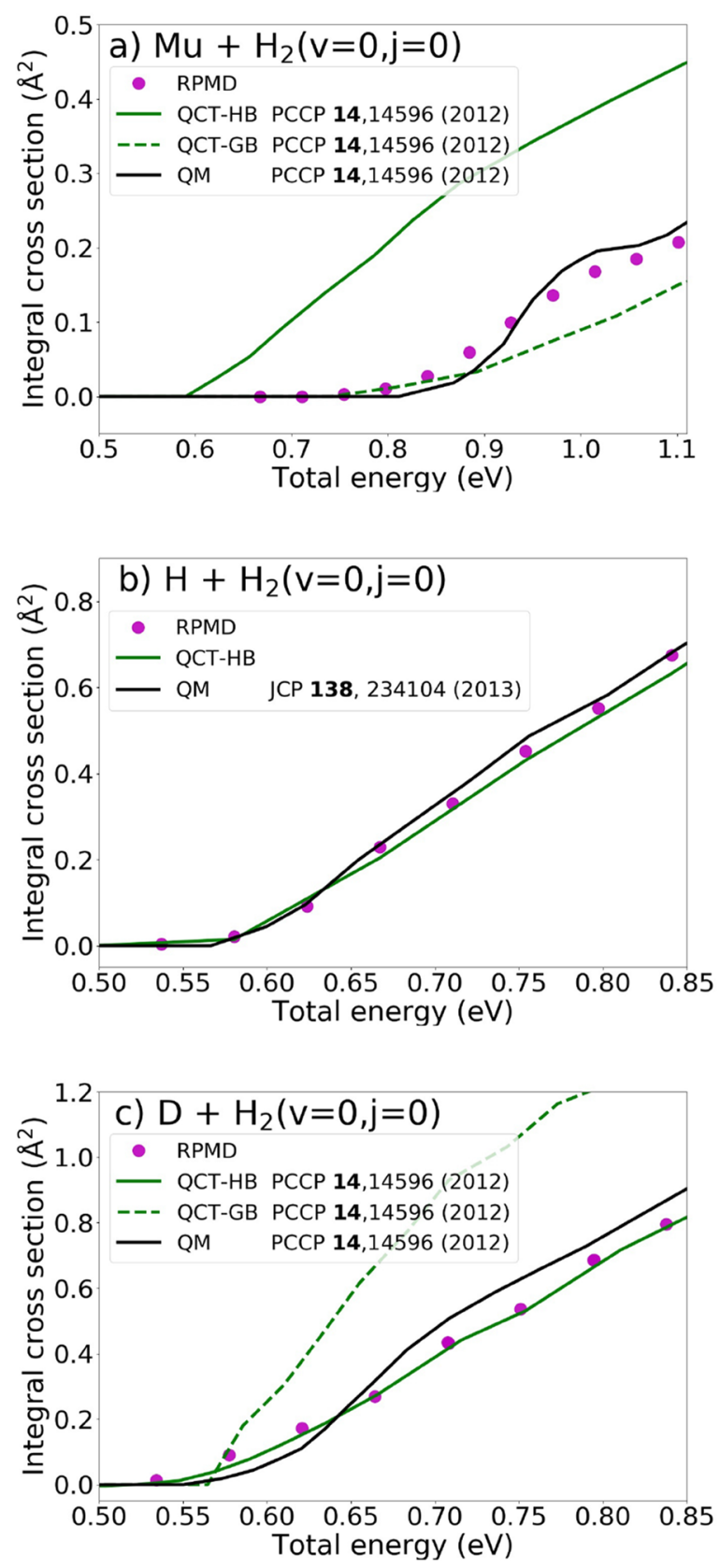

FIG. 1. Integral cross sections for the reaction of $M u / H / D+\mathrm{H}_{2}(v=0)$ calculated using RPMD. Reference quantum dynamics results and QCT results are taken from Refs. 44 and 59. 
simulations due to the absence of any tunneling in the QCT simulations. ${ }^{44}$ We employ the BKMP2 PES, ${ }^{58}$ a modified velocity-Verlet integrator with a time step of $0.02 \mathrm{fs}, 40000$ trajectories per collision energy, $\beta_{-}=300$ a.u., and a ratio of $\frac{n}{\beta \omega_{\mathrm{H}_{2}}}>4$, which guarantees convergence with the number of beads. $d_{\min }$ is set to $1.078 \mathrm{bohr}$. The QCT distance between $\mathrm{A}$ and $\mathrm{BC}$ is $d(A, B C)_{\mathrm{QCT}}=12$ bohr. Note
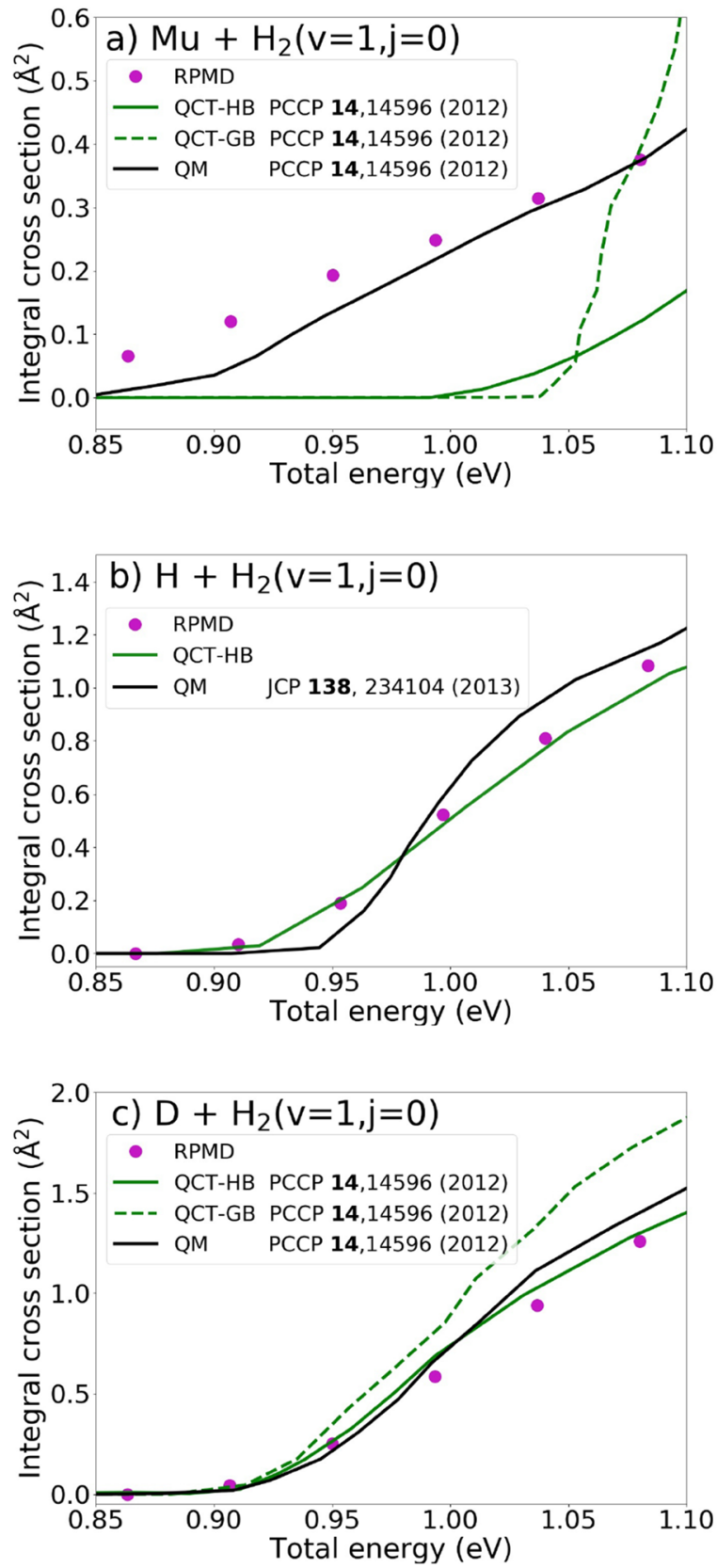

FIG. 2. Integral cross sections for the reaction of $M u / H / D+H_{2}(v=1)$ calculated using RPMD. Reference quantum dynamics results and QCT results are taken from Refs. 44 and 59. that the maximal impact parameter is typically slightly higher in the RPMD simulations than in the QCT simulations. However, at low collision energies close to the classical threshold, it can be up to a factor of 1.5 higher than in the QCT simulations.

First, we focus on the reactivity of $\mathrm{Mu} / \mathrm{H} / \mathrm{D}$ with $\mathrm{H}_{2}$ in its vibrational ground state. Figure 1 presents the integral cross section of the $\mathrm{Mu} / \mathrm{H} / \mathrm{D}+\mathrm{H}_{2}(v=0)$ reactions. For the $\mathrm{Mu}+\mathrm{H}_{2}$ reaction [panel (a)], it is shown that the proposed RPMD scattering approach very well reproduces the exact quantum mechanical (QM) calculations from Ref. 44. The straight forward QCT calculations employing histogram binning (HB), i.e., treating every trajectory equally, however, show a much lower threshold for reactivity compared to the QM calculations due to leakage of ZPE, which is well documented in the literature. ${ }^{44}$ Note that this problem can partly be circumvented employing Gaussian binning (GB), ${ }^{60,61}$ as shown in Fig. 1(a). GB diminishes the contribution to the cross section for trajectories that violate the ZPE condition, i.e., where the resulting diatom has less internal energy than required from the quantum mechanical ZPE. Yet, it can be seen that the GB results underestimate the cross sections away from the threshold, whereas the proposed approach closely reproduces the exact results. In panels (b) and (c) of Fig. 1, it can be seen that RPMD can reproduce the QM integral cross sections for the $\mathrm{H}+\mathrm{H}_{2}(v=0)$ and $\mathrm{D}+\mathrm{H}_{2}(v=0)$ reactions very well. For these two cases, the QCT simulations also perform well.

Second, we investigate the integral cross sections for the same reactions but employing vibrationally excited $\mathrm{H}_{2}, v=1$. The resulting cross sections are displayed in Fig. 2. Again, the proposed RPMD scattering approach very well reproduces all QM integral cross sections. For this case, both QCT variants (HB and GB) cannot reproduce the exact $\mathrm{QM}$ results for the $\mathrm{Mu}+\mathrm{H}_{2}$ reaction and display a higher threshold for reactivity. This is due to the missing tunneling contributions through the $v=1$ adiabatic barrier in the QCT calculations. ${ }^{44}$ This problem is mitigated in the RPMD simulations, as some quantum contributions are included due to the finite extension of the ring polymers.

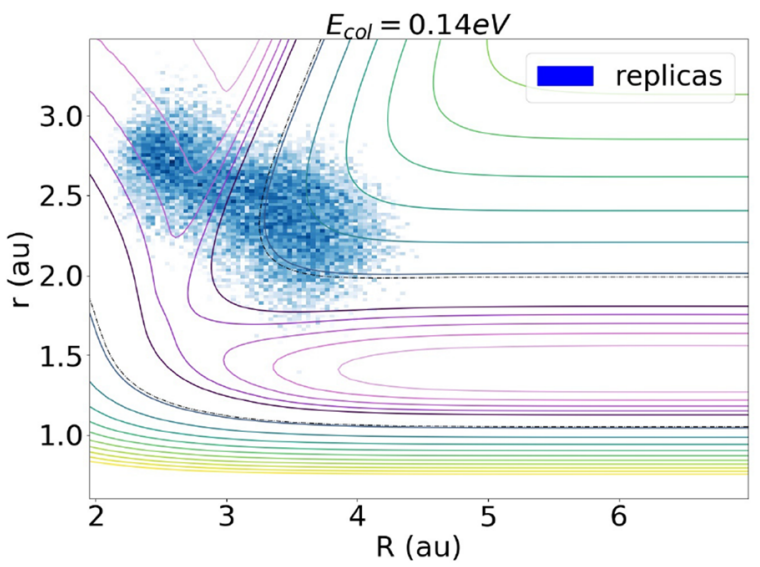

FIG. 3. Density of the ring polymer beads for the $\mathrm{Mu}+\mathrm{H}_{2}(v=1)$ reaction for reactive trajectories at the time of barrier crossing at $E_{\text {col }}=0.14 \mathrm{eV}$ and for impact parameters $b<3$ a.u. $r$ refers to the $\mathrm{H}_{2}$ distance and $R$ to the distance of Mu to the center of mass of $\mathrm{H}_{2}$. The PES for the system is plotted for comparison with the remaining coordinated relaxed. 
The classically forbidden contributions in RPMD can be best seen by inspecting plots of the ring polymer density during the barrier crossing given in Fig. 3. Figure 3 displays the density of the beads for all reactive ring polymer trajectories for a collision energy of $0.14 \mathrm{eV}$ and impact parameters $b<3$ a.u. at about the time of the barrier crossing. It can be clearly seen that the ring polymer "cuts the corner" through a higher energy region to allow for reactivity. This region is never reached by QCT trajectories due to insufficient energy in the system, yet in the extended ring polymer phase space, it is possible as the ring polymer is never completely located in the higher energy region, but some beads are always in the lower energy regions on both sides of the barrier, which lowers the effective (i.e., averaged) energy of the full ring polymer when corner cutting.

\section{CONCLUSION}

In this manuscript, we focused on the description of triatomic systems and a first approach to combine QCT with RPMD for state-selective scattering simulations, and many generalizations and extensions of the approach have to be left for future work. First of all, the current approach to initiate the vibrational excitation is described for triatomics but is readily generalized to any polyatomic system including gas-surface reactions. Even better initial energies are obtained in QCT by employing the adiabatic switching procedure. This procedure cannot be generalized to the present approach right away and has to be left for further development. Furthermore, the initialization of distinct rotational or ro-vibrational states and the analysis of distinct ro-vibrational final states to obtain state-to-state cross sections have to be left for future work. Finally, non-adiabatic effects can be important in many bimolecular reactions. ${ }^{62,63}$ Several approaches have been proposed to include the treatment of nonadiabatic effects within RPMD. ${ }^{64-66}$ It has to be explored which of these approaches performs best for reactive scattering problems. The presented approach for choosing $\beta$ in the current context of microcanonical RPMD and initial state-specificity is a first attempt, which can be reasonably motivated and provides good results for integral cross sections. However, more detailed investigation of its reliability and robustness as well as the investigation of other approaches for choosing $\beta$ should be pursued. To this end, one can, for example, learn from the attempt to tune $\beta$ for the computation of microcanonical rates for the one-dimensional Eckart model potential. ${ }^{57}$ While RPMD rate theory is the preferred, much more efficient, and direct way to obtain thermal rate constants, we think that, due to good agreement with the exact quantum simulations, one can expect to also obtain reasonable thermal rate constants from calculations following our approach. However, as we have to introduce several additional steps compared to standard RPMD rate theory (e.g., nonequilibrium initial conditions), the results will most likely not match perfectly.

In conclusion, we have presented an efficient way to obtain initial state-selective cross sections for bimolecular gas phase and gas-surface reactions. The proposed approach combines the ideas of quasiclassical trajectories with the ring polymer molecular dynamics method. We showed that this combination is possible and can alleviate several problems intrinsic to the QCT approach, i.e., the problem of ZPE constraints and the neglect of tunneling contributions, which was discussed employing the prototypical $\mathrm{Mu} / \mathrm{H} / \mathrm{D}$
$+\mathrm{H}_{2}(v=0,1)$ reactions. The new approach is easily implemented, is numerically efficient, and significantly improves the accuracy of QCT simulations.

\section{DATA AVAILABILITY}

The data that support the findings of this study are available from the corresponding author upon reasonable request.

\section{REFERENCES}

${ }^{1}$ H. Chadwick and R. D. Beck, Annu. Rev. Phys. Chem. 68, 39 (2017).

${ }^{2}$ P. M. Hundt, B. Jiang, M. E. van Reijzen, H. Guo, and R. D. Beck, Science 344, 504 (2014).

${ }^{3}$ A. J. Orr-Ewing, J. Chem. Phys. 140, 090901 (2014).

${ }^{4}$ R. I. Kaiser, D. S. Parker, and A. M. Mebel, Annu. Rev. Phys. Chem. 66, 43 (2015).

${ }^{\mathbf{5}}$ N. Balucani, F. Leonori, and P. Casavecchia, Energy 43, 47 (2012).

${ }^{6}$ S. Yan, Y.-T. Wu, B. Zhang, X.-F. Yue, and K. Liu, Proc. Natl. Acad. Sci. U. S. A. 105, 12667 (2008).

${ }^{7}$ F. Wang, J.-S. Lin, and K. Liu, Science 331, 900 (2011).

${ }^{8}$ W. Shiu, J. Lin, and K. Liu, Phys. Rev. Lett. 92, 103201 (2004).

${ }^{9}$ W. Zhang, H. Kawamata, and K. Liu, Science 325, 303 (2009).

${ }^{10}$ B. L. Yoder, R. Bisson, and R. D. Beck, Science 329, 553 (2010).

${ }^{11}$ K. Liu, Annu. Rev. Phys. Chem. 67, 91 (2016).

${ }^{12}$ P. Maroni, D. C. Papageorgopoulos, M. Sacchi, T. T. Dang, R. D. Beck, and T. R. Rizzo, Phys. Rev. Lett. 94, 246104 (2005).

${ }^{13}$ D. R. Killelea, V. L. Campbell, N. S. Shuman, and A. L. Utz, Science 319, 790 (2008).

${ }^{14}$ G. Schiffel and U. Manthe, J. Chem. Phys. 132, 191101 (2010).

${ }^{15}$ R. Welsch and U. Manthe, J. Phys. Chem. Lett. 6, 338 (2015).

${ }^{16}$ R. Welsch and U. Manthe, J. Chem. Phys. 141, 051102 (2014).

${ }^{17}$ R. Welsch and U. Manthe, J. Chem. Phys. 141, 174313 (2014).

${ }^{18}$ R. Ellerbrock and U. Manthe, Chem. Phys. 482, 106 (2017).

${ }^{19}$ R. Ellerbrock and U. Manthe, J. Chem. Phys. 147, 241104 (2017).

${ }^{20}$ R. Ellerbrock and U. Manthe, J. Chem. Phys. 148, 224303 (2018).

${ }^{21}$ W. H. Miller, S. D. Schwartz, and J. W. Tromp, J. Chem. Phys. 79, 4889 (1983).

${ }^{22}$ D. H. Zhang and J. C. Light, J. Chem. Phys. 104, 6184 (1996).

${ }^{23}$ F. Matzkies and U. Manthe, J. Chem. Phys. 106, 2646 (1997).

${ }^{24}$ U. Manthe, Mol. Phys. 109, 1415 (2011).

${ }^{25}$ R. Welsch, F. Huarte-Larrañaga, and U. Manthe, J. Chem. Phys. 136, 064117 (2012).

${ }^{26}$ R. Welsch and U. Manthe, Mol. Phys. 110, 703 (2012).

${ }^{27}$ U. Manthe and R. Welsch, J. Chem. Phys. 140, 244113 (2014).

${ }^{28}$ H.-D. Meyer, U. Manthe, and L. S. Cederbaum, Chem. Phys. Lett. 165, 73 (1990).

${ }^{29}$ U. Manthe, H. D. Meyer, and L. S. Cederbaum, J. Chem. Phys. 97, 3199 (1992).

${ }^{30} \mathrm{H}$. Wang and M. Thoss, J. Chem. Phys. 119, 1289 (2003).

${ }^{31}$ U. Manthe, J. Chem. Phys. 128, 164116 (2008).

${ }^{32}$ U. Manthe, J. Chem. Phys. 105, 6989 (1996).

${ }^{33}$ U. Manthe, J. Chem. Phys. 130, 054109 (2009).

${ }^{34}$ F. Gatti, B. Lasorne, H.-D. Meyer, and A. Nauts, Applications of Quantum Dynamics in Chemistry (Springer, 2017), Vol. 98.

${ }^{35}$ R. Welsch and U. Manthe, J. Chem. Phys. 137, 244106 (2012).

${ }^{36}$ R. Welsch and U. Manthe, J. Chem. Phys. 138, 164118 (2013).

${ }^{37}$ R. Welsch and U. Manthe, J. Chem. Phys. 142, 064309 (2015).

${ }^{38}$ G. Schiffel and U. Manthe, J. Chem. Phys. 132, 084103 (2010).

${ }^{39}$ M. Karplus, R. N. Porter, and R. Sharma, J. Chem. Phys. 43, 3259 (1965).

${ }^{40}$ R. N. Porter, Annu. Rev. Phys. Chem. 25, 317 (1974).

${ }^{41}$ F. J. Aoiz, V. J. Herrero, and V. Sáez Rábanos, J. Chem. Phys. 97, 7423 (1992).

${ }^{42}$ U. Lourderaj, R. Sun, S. C. Kohale, G. L. Barnes, W. A. de Jong, T. L. Windus, and W. L. Hase, Comput. Phys. Commun. 185, 1074 (2014). 
${ }^{43}$ P. Jambrina, E. García, V. J. Herrero, V. Sáez-Rábanos, and F. J. Aoiz, J. Chem. Phys. 135, 034310 (2011).

${ }^{44}$ P. Jambrina, E. García, V. J. Herrero, V. Sáez-Rábanos, and F. J. Aoiz, Phys. Chem. Chem. Phys. 14, 14596 (2012).

${ }^{45}$ I. R. Craig and D. E. Manolopoulos, J. Chem. Phys. 121, 3368 (2004).

${ }^{46}$ S. Habershon, D. E. Manolopoulos, T. E. Markland, and T. F. Miller, Annu. Rev. Phys. Chem. 64, 387 (2013).

${ }^{47}$ Y. V. Suleimanov, J. W. Allen, and W. H. Green, Comput. Phys. Commun. 184, 833 (2013).

${ }^{48}$ Y. V. Suleimanov, F. J. Aoiz, and H. Guo, J. Phys. Chem. A 120, 8488 (2016).

${ }^{49}$ S. Habershon and D. E. Manolopoulos, J. Chem. Phys. 131, 244518 (2009).

${ }^{50} \mathrm{Q}$. Liu, L. Zhang, Y. Li, and B. Jiang, J. Phys. Chem. Lett. 10, 7475 (2019).

${ }^{51}$ R. Pérez de Tudela, F. J. Aoiz, Y. V. Suleimanov, and D. E. Manolopoulos, J. Phys. Chem. Lett. 3, 493 (2012).

${ }^{52}$ Y. V. Suleimanov, R. P. de Tudela, P. G. Jambrina, J. F. Castillo, V. SáezRábanos, D. E. Manolopoulos, and F. J. Aoiz, Phys. Chem. Chem. Phys. 15, 3655 (2013).
${ }^{53}$ I. R. Craig and D. E. Manolopoulos, J. Chem. Phys. 122, 084106 (2005).

${ }^{54}$ I. R. Craig and D. E. Manolopoulos, J. Chem. Phys. 123, 034102 (2005).

${ }^{55}$ R. Welsch, K. Song, Q. Shi, S. C. Althorpe, and T. F. Miller III, J. Chem. Phys. 145, 204118 (2016).

${ }^{56}$ T. J. H. Hele, M. J. Willatt, A. Muolo, and S. C. Althorpe, J. Chem. Phys. 142, 134103 (2015).

${ }^{57}$ X. Tao, P. Shushkov, and T. F. Miller, J. Chem. Phys. 152, 124117 (2020).

${ }^{58}$ A. I. Boothroyd, W. J. Keogh, P. G. Martin, and M. R. Peterson, J. Chem. Phys. 95, 4343 (1991).

${ }^{59}$ B. Jiang and H. Guo, J. Chem. Phys. 138, 234104 (2013).

${ }^{60}$ L. Bonnet and J. Rayez, Chem. Phys. Lett. 277, 183 (1997).

${ }^{61}$ L. Bonnet and J.-C. Rayez, Chem. Phys. Lett. 397, 106 (2004).

${ }^{62}$ L. Che, Z. Ren, X. Wang, W. Dong, D. Dai, X. Wang, D. H. Zhang, X. Yang, L. Sheng, G. Li et al., Science 317, 1061 (2007)

${ }^{63}$ J. Palma and U. Manthe, J. Chem. Phys. 146, 214117 (2017).

${ }^{64}$ J. O. Richardson and M. Thoss, J. Chem. Phys. 139, 031102 (2013).

${ }^{65}$ P. Shushkov, R. Li, and J. C. Tully, J. Chem. Phys. 137, 22 A549 (2012).

${ }^{66}$ X. Tao, P. Shushkov, and T. F. Miller, J. Chem. Phys. 148, 102327 (2018). 